\title{
Effectiveness of telehealth in preventing coronavirus disease in patients with HIV
}

\author{
Moradali Zareipour ${ }^{1}$, Ehsan M. Ehsan ${ }^{2}$ \\ ${ }^{1}$ Health System Research Unit, Health Center of Urmia, Urmia University of Medical sciences, Urmia, Iran \\ ${ }^{2}$ Department of Public Health, School of Public Health, Jiroft University of Medical Sciences, Jiroft, Iran
}

HIV AIDS Rev 2021; 20, 3: 229-230

DOI: https://doi.org/10.5114/hivar.2021.109256

Prescription of medications for human immunodeficiency virus (HIV) can help people with HIV (PWHIV) to live longer and healthier lives and reduce the risk of transmitting HIV to their partner [1]. In this regard, development of new solutions is vital to strengthen patient-physician communication and maintain HIV care in controlling the HIV epidemic in Iran. According to studies conducted in Iran and the United States, the challenges of receiving medication, counseling, and maintaining adherence to medications were identified as: transportation costs, long distances, unemployment, homelessness, depression, low income, and social stigma $[2,3]$. Currently, we are facing the coronavirus disease 2019 (COVID-19) pandemic, which has disrupted the delivery of health services to the target population, especially to HIV patients worldwide [4]. The number of COVID-19 cases has increased significantly compared to severe acute respiratory syndrome and Middle East respiratory syndrome. Furthermore, decrease of the disease activity is not probable, which implies that the controlling measures must be in place for a longer period of time [5]. Considering social distance and staying at home as the cornerstone for prevention of COVID-19 [6], providing appropriate services to HIV patients and helping them to have timely drug adherence have highlighted the need for application of telecommunication technologies.

In telehealth, health care workers communicate with clients or patients using cameras, equipment, video conferencing, telephones, and videos to evaluate, plan, intervene, evaluate the results of nursing care, provide counseling, and educate on health. Telehealth is an acceptable and easy alternative to rendering prevention and treatment services to patients. Furthermore, patients spend less time for a visit since they can stay at home and should not commute to the health

Address for correspondence: Dr. Ehsan Movahed Ehsan, Department of Public Health, School of Public Health, Jiroft University of Medical Sciences, Jiroft, Iran,

e-mail: ehsanmovahed89@yahoo.com

centers. Other family members may also be encouraged to check on their health. The visit is completely informal and the patients are more relaxed. In addition, patients who often forget to bring their medicine bottles to the doctor's office or do not remember the medicines' names can easily show their medicines through cyberspace. In a study by Dandachi et al., many PWHIV reported avoiding high costs and COVID-19 as well as feeling comfortable in application of the telehealth system. Following the telehealth visit, more than $90 \%$ of these patients were at equal satisfaction levels considering use of services provided by the telehealth system and physical face-to-face visits [3].

Numerous studies have reported promising aspects of telehealth, such as online-based sexual health access to promote HIV testing and to address specific interventions among PWHIV, including smoking cessation, depression treatment, viral suppression, and high patient satisfaction. Based on the literature, telehealth can be an acceptable, safe, practical, cost-effective, and efficient alternative to referring to health centers for HIV management [7].

Social media, such as mobile apps, are especially important for patients' access to preventive, screening, and monitoring services, telemonitoring of medical vital signs, provision of preventive and therapeutic recommendations such as using masks, observing social distance, and raising patients' awareness about COVID symptoms and the ways to deal with them. With regard to PWHIV, social media and mobile apps can be used to engage and protect PWHIV and at-risk individuals. To this end, virtual groups can be formed to identify PWHIV, monitor their medication adherence status closely, and provide them with the required recommendations. Considering the social stigma and discrimination due to HIV, PWHIV are not willing to be identified, so they

Article history:

Received: 02.01.2021

Received in revised form: 10.03.2021

Accepted: 11.03.2021

Available online: 30.08 .2021
International Journal of HIV-Related Problems

HIV \& AIDS Re $v$ i e w 
do not apply to receive the medicines. In this regard, many patients can be identified and followed through telehealth as an effective approach. This facilitates reaching the goals of 909090 set by the World Organization in 2030.

The following measures may be useful for preventing and controlling COVID-19 in HIV patients using telehealth:

- Providing the remote care tools based on specific needs, not in general; the confidentiality of care should be maintained.

- Developing and implementing electronic methods that can remove stigma and discrimination from HIV patients.

- Providing the patients with a dedicated SIM card for internet connection.

- Assigning some people to deliver the medicines to the patients.

- Providing medication instructions in a simple and fluent way using the native language for illiterate people through tele-education.

- Motivating people with poor drug adherence through teleeducation.

- Allocating grants to patients to buy computers and smartphones.

- Informing patients about the existence of remote care tools in virtual networks.

- Holding remote workshops on health for healthcare workers.

- Creating remote care tools at a lower cost with easier application.

In conclusion, the demand for telehealth services has increased in the developed countries and health centers have turned to telehealth service delivery approaches. Research results have shown that telehealth was as efficient as or even more efficient than personal treatments with regard to many diseases. Application of telehealth not only is effective in treating diseases, but also provides long-term access and reduces costs. As a result, many developing countries have been encouraged to provide remote health care services and use new communication technologies. Given the current prevalence of COVID-19, health authorities are recommended to use telehealth therapy to provide effective health care services to HIV patients.

\section{Conflict of interest}

The authors have no conflict of interest.

\section{References}

1. Bavinton BR, Pinto AN, Phanuphak N, et al. Viral suppression and HIV transmission in serodiscordant male couples: an international, prospective, observational, cohort study. Lancet HIV 2018; 5: e438-e447.

2. Morowatisharifabad M, Movahed E, Farokhzadian J, et al. Depression and physical activity among HIV-positive people: integrated care towards 90-90-90. HIV AIDS Rev 2019; 18: 131-136.

3. Dandachi D, Freytag J, Giordano TP, Dang BN. It is time to include telehealth in our measure of patient retention in HIV care. AIDS Behav 2020. DOI: 10.1007/s10461-020-02880-8 [Epub ahead of print].

4. Lipsitch M, Swerdlow DL, Finelli L. Defining the epidemiology of Covid-19 - studies needed. N Engl J Med 2020; 382: 1194-1196.
5. Kelly-Cirino C, Mazzola LT, Chua A, Oxenford CJ, Van Kerkhove MD.An updated roadmap for MERS-CoV research and product development: focus on diagnostics. BMJ Global Health 2019; 4 (Suppl 2): e001105.

6. Zareipour M, Jadgal MS, Movahed E. Health ambassadors role in self-care during COVID-19 in Iran. Journal Mil Med 2020; 22: 672-674.

7. Brennan DJ, Souleymanov R, Lachowsky N, Betancourt G, Pugh D, McEwen O. Providing online-based sexual health outreach to gay, bisexual, and queer men in Ontario, Canada: qualitative interviews with multisectoral frontline service providers and managers. AIDS Patient Care STDS 2018; 32: 282-287. 